\title{
DEVO-DARWINISMO. LO QUE EL LENGUAJE NOS ENSEÑA SOBRE EL PAPEL DEL DESARROLLO EN LA EVOLUCIÓN NATURAL
}

\section{DEVO-DARWINISM. WHAT LANGUAGE TELLS US ABOUT THE ROLE OF DEVELOPMENT ON EVOLUTION}

\author{
Guillermo LORENZO* \\ Dpto. de Filología Española (Área de Lingüistica General) \\ Universidad de Oviedo
}

\begin{abstract}
RESUMEN: En su intento por neutralizar la aparente excepcionalidad del lenguaje, Darwin intuyó un hilo de continuidad entre este rasgo exclusivo de los humanos y el canto de los pájaros. Recientes hallazgos moleculares confirman que pudiera tratarse de un ejemplo de verdadera homología. Este trabajo repasa este aspecto del pensamiento de Darwin y comenta su significado en el contexto de la moderna biología evolutiva del desarrollo. Se sostiene que la confirmación de la mencionada homología no requiere, sin embargo, aceptar la existencia de una línea de evolución gradual entre una y otra habilidad, ni que en el proceso hayan realmente operado los mecanismos de selección sexual o natural.
\end{abstract}

Palabras Clave: Cognición animal, Darwin, evo-devo, homología, lenguaje,

ABSTRACT: Darwin established an evolutionary link between birdsong and human language when trying to coun-

* e-mail: glorenzo@uniovi.es 
teract the apparent exceptionality of the latter, and recent molecular findings have confirmed that it might be an example of true homology. This essay reviews this aspect of Darwin's thought and comments its significance within the context of current evolutionary developmental biology. It nevertheless contends that the corroboration of this homology does require neither the existence of a gradual path of evolution between both abilities or the guidance by the mechanisms of sexual or natural selection.

KEYwORDS: Animal cognition, Darwin, evo-devo, homology, language.

\section{Introducción*}

Darwin puso un especial empeño en tratar de relativizar la idea de que los humanos posean características sin equivalente alguno en el resto del mundo orgánico. Los numerosos pasajes que dedicó en su obra al lenguaje, especialmente en The descent of man (1871), tienen que ver con este propósito. En concreto, Darwin planteó que el lenguaje podría estar emparentado evolutivamente con otras formas de expresividad vocal relacionadas con la función reproductiva en diversas especies. Apuntó, asimismo, que el canto de los pájaros probablemente fuese la habilidad animal con la que el lenguaje humano muestra más semejanzas y defendió que los primeros humanos, o sus más inmediatos ancestros, debieron poseer una facultad semejante y emparentada con aquella que evolucionó gradualmente hasta dar lugar al lenguaje tal cual hoy lo conocemos. De este modo, una característica especialmente desafiante para sus ideas, por su aparente especificidad humana, quedaba sometida al principio de continuidad orgánica, acaso la piedra angular de sus modelos de evolución mediante selección natural y sexual. La primera parte de este artículo contextualiza y presenta con cierto detalle las ideas de Darwin relativas a esta cuestión y la segunda parte las relaciona con algunos hallazgos moleculares recientes que apuntan a que la afinidad entre el canto de los pájaros y el lenguaje humano podría corresponderse con un

* Este trabajo ha sido realizado al amparo del proyecto de investigación «Biolingüística: fundamento genético, desarrollo y evolución del lenguaje» (HUM2007-60427/FILO), subvencionado por el Ministerio de Ciencia e Innovación con financiación parcial FEDER. 
caso de homología real. La tercera y última sección aclara que dicha homología remite en último término a los mecanismos subyacentes a la diferenciación y la individualización durante el desarrollo de las estructuras neuroanatómicas en que se basa el ejercicio de las respectivas habilidades. En ella se argumenta, además, que desde la perspectiva de la moderna biología evolutiva del desarrollo un hilo de continuidad como el intuido por Darwin no nos obliga sin embargo a asumir un modelo de evolución estrictamente gradual ni estrictamente acorde con una lógica adaptacionista. Por el contrario, la pauta de variación muy restringida y sujeta a discontinuidades cualitativas entre las habilidades con que la inspiración de Darwin nos invita a relacionar el lenguaje parece mucho más consonante con un modelo en que la evolución se ve sujeta a las fuertes constricciones que operan sobre el desarrollo y en el que cobran especial protagonismo episodios esporádicos de cambio abrupto.

\section{La facultad del lenguaje: Humana, pero no tanto}

La auto-conciencia de su singularidad dentro del reino animal puede razonablemente contarse entre las señas de identidad que caracterizan a la especie humana. Su reflejo en la taxonomización del mundo orgánico con anterioridad a Darwin ha sido siempre evidente, aunque diversa la distancia con que diferentes autores han marcado la brecha entre el hombre y las restantes formas de la vida sobre la tierra. Linneo clasificó a los humanos como un "género» dentro de los Primates, pero desde entonces las más renombradas figuras de la biología pre-darwinista tendieron a acentuar aún más la distancia: Cuvier los elevó a la categoría de «orden» (Bimana), Owen a la de «subclase» (Archencephala) e I. Geoffroy St-Hilaire a la de «reino», es decir, como una entidad aparte y en igualdad de rango con el resto de los Animalia ${ }^{1}$. En todos los casos, la consideración de las características del cerebro y de las facultades mentales del hombre relativamente a las de cualquier otra especie ha estado en la base de la acentuación de

${ }^{1}$ Caroli Linnaei, Systema naturae per regna tria naturae, secundum classes, ordines, genera, species, cum characteribus, differertiis, synonymis, locis (1735-70); Georges Cuvier, Le règne animal distribué d'après son organisation, pour servir de base à l'histoire naturelle des animeaux et d'introduction à l'anatomie compareé (1817); Richard Owen, On the classification and geographical distribution of the Mammalia (1859); Isidore Geoffroy St-Hilaire, Histoire naturelle générale des règnes organiques (1854-62). 
esa brecha. Para algunos autores, ejemplarmente Gratiolet u Owen², tanto la morfología cerebral como las capacidades mentales asociadas a ella eran factores que, dada su relación directa (causa-efecto), debían ser considerados por igual en la caracterización biológica de los humanos; en cambio para otros, como Wagner, Quatrefages o Huxley ${ }^{3}$, siendo las diferencias morfológicas menores o, en todo caso, no cualitativas, eran las facultades mentales más distintivas del hombre las que verdaderamente debían pesar en su declaración como una especie singular. Con independencia del debate sobre la especificidad de la conformación del cerebro humano (realmente agrio, al menos el que enfrentó personalmente a Owen y Huxley $)^{4}$, lo cierto es que todas las partes coincidieron en considerar únicas muchas de las capacidades mentales del hombre y, entre ellas, la facultad humana del lenguaje ${ }^{5}$. Este es, muy sucintamente relatado, el contexto que explica que Darwin decidiera dedicar algunas páginas de sus principales trabajos a tratar de clarificar evolutivamente la posesión por parte del hombre de una facultad lingüística con características aparentemente únicas.

Desde un punto de vista estrictamente morfológico, Darwin sostuvo a propósito de la supuesta singularidad del cerebro humano básicamente las posiciones previamente defendidas por $\mathrm{Huxley}^{6}$. Los principales parámetros anatómicos en que se centró la discusión sobre el tema fueron el tamaño del cerebro en términos absolutos y relativos al del resto del cuerpo, el número y profundidad

${ }^{2}$ Louis Pierre Gratiolet, Mémoire sur les plis cérébraux (1854); Richard Owen, On the anatomy of vertebrates (1866-68).

3 Rudolph Wagner, Vorstudien zu einer wissenschaftlinchen Morphologie und Physiologie des menschlichen Gehirns als Seelenorgan (1860-62); Jean-Louis-Armand de Quatrefages, L'Espèce humaine (1861); Thomas Henry Huxley, Evidence as to man's place in nature (1863).

${ }^{4}$ Véase Rupke (1994: 270-286).

5 Gratiolet, por ejemplo, hablaba del reino humano como del «règne de verbe» (Anatomie comparée du système nerveux, 1839-57, vol.II), Owen destacaba entre las capacidades intelectuales humanas «the power of framing general propositions, and of expressing thought in articulate speech» (Medical Times and Gazette, 1862 (II), 373; citado por Rupke 1994) y Wagner apuntaba al lenguaje como «símbolo» de la capacidad de pensamiento abstracto que da lugar a las nociones humanas de moralidad y divinidad (Der Kampf um die Seele, 1857). El célebre obispo Wilberforce se refería al «man's power of articulate speech» como una de las bases de la supremacía humana sobre el resto de la tierra («Darwin’s Origin of Species», Quaterly Review vol.108, 1860).

${ }^{6}$ De hecho, Darwin incorporó a la segunda versión de The descent of man (1874) el texto de Huxley "Note on the resemblances and differences in the structure and the development of the brain in man and apes» como apéndice a la Parte I. 
de los surcos y la conformación de ciertas estructuras cerebrales como la curvatura posterior de los ventrículos laterales de ambos hemisferios y la presencia en cada uno de estos de un «hipocampo menor». Huxley sostuvo, con relación a todos ellos, que la comparación del cerebro humano con el de otros simios demostraba que se trataba sin excepción de rasgos sujetos a diferencias de tipo exclusivamente cuantitativo, de modo que ninguna característica cerebral debería ser considerada como específicamente humana. Darwin suscribió plenamente la opinión de que los humanos disponen de un cerebro más desarrollado que el de otras especies (Darwin 1871, 22-23), no un tipo diferente de cerebro, y recurrió también para apoyarla al argumento del desarrollo: un cerebro humano muestra, a los siete meses, las características del cerebro de un babuino adulto (Darwin $1871,26-28)^{7}$. Se aseguró, además, de acreditar la existencia de un cierto margen de variación cuantitativa entre los cerebros humanos, ingrediente fundamental, como es bien sabido, para que la receta evolutiva de la selección natural pueda funcionar (Darwin 1871, 196). Desde el punto de vista de las facultades mentales, Darwin expresó en cambio opiniones más próximas a las de Owen que a las de Huxley, en el sentido de dar por supuesta una estrecha conexión entre el grado de desarrollo alcanzado por el cerebro y las capacidades mentales asociadas a éste. Defendió incluso que la propia variación intraespecífica observable en los humanos podía ponerse en relación con diferencias en las aptitudes mentales de unos u otros, llegando incluso a establecer generalizaciones de tinte racista sobre las capacidades de las diferentes poblaciones humanas y sobre la superioridad de los británicos (Darwin 1871, 74-75)

Darwin consideró además que esta conexión entre el grado de desarrollo y las capacidades cerebrales podía particularizarse para el caso de las funciones mentales superiores, aparentemente exclusivas y por tanto más desafiantes para su enfoque. También, naturalmente, para el caso del lenguaje, en cuyo caso Darwin habló de una relación bidireccional: por una parte, un alto grado de desarrollo cerebral fue sin duda un factor facilitador para la existencia de una facultad lingüística altamente compleja como la humana; por otra parte, la propia posesión y ejercicio de esta facultad pudo haber incidido en un incremento del desarrollo cerebral humano (Darwin 1871, 110). Con todos estos elementos

7 Darwin basa la observación en Theodor Ludwig Wilhem Bischoff, «Die Grosshirnwindungen des Menschen» (1868).

${ }^{8}$ Matizadas, eso sí, por la remisión del dato a James Cowles Prichard. 
debidamente establecidos, lo único que necesitaba Darwin para acomodar a sus explicaciones un rasgo tan excepcional en apariencia como el lenguaje era la constatación de la existencia de formas de lenguaje, proto-lenguaje o cuasi-lenguaje correspondientes a grados de desarrollo cerebral inferiores al humano. Por tanto, demostrar la no excepcionalidad y relativizar la especificidad de la facultad del lenguaje propia del ser humano.

Darwin encontró ese hilo de continuidad en el canto de los pájaros, que consideraba, a su vez, un terreno ideal para la aplicación del mecanismo evolutivo de «selección sexual», es decir, la proliferación de los rasgos de los organismos más aventajados en el acceso a parejas y en la consumación de contactos reproductivos ${ }^{9}$. En una rápida síntesis, Darwin propuso que el lenguaje evolucionó gradualmente a partir de primitivas formas de vocalización empleadas por los primeros humanos o por algún antepasado próximo del hombre como reclamo sexual, las cuales serían una versión evolucionada y emparentada con tantas otras manifestaciones de este tipo de comportamiento, como el canto de los pájaros, ampliamente extendidas en el mundo natural. De esta manera, Darwin conseguía someter el lenguaje a todos y cada uno de los principios fundamentales de su modelo: continuismo, adaptacionismo (en este caso en relación con la función reproductiva) y gradualismo. Interesa reconstruir su argumento con algún detalle.

Darwin pensó, en primer lugar, que la suposición de que el lenguaje sea un resultado más de la diversificación de una capacidad ancestral de tipo musical es mucho más razonable que su contraria, es decir, que la música sea una característica humana de algún modo derivada del lenguaje. La justificación es simple: mientras que el lenguaje es una característica distintiva de los humanos, la práctica y el sentido de la musicalidad se encuentra en cambio ampliamente exten-

9 Aunque la explicación evolutiva del lenguaje fue un tema tabú para la lingüística teórica prácticamente hasta la última década del siglo XX, una interesante excepción es el caso del lingüista danés Otto Jepersen, quien además hizo propias las tesis darwinistas sobre la relación evolutiva entre el canto de algunos animales y el lenguaje y sobre la motivación sexual de las más primitivas formas de lenguaje. Véase Jespersen (1922). Con diferentes matices, la aplicación del mecanismo de selección sexual al caso del lenguaje ha sido defendida recientemente en Ridley (1993), Deacon (1997), Miller (2000), Okanoya (2002), Burling (2005) y Mithen (2006). Véase Lorenzo (2006: Capítulo 3) a propósito de las limitaciones de la idea para explicar el diseño del lenguaje como resultado de la evolución natural. 
dida en el reino animal ${ }^{10}$. En segundo lugar, la presencia en el lenguaje de propiedades de tipo musical (como el carácter discreto de las señales o el empleo de la tonalidad y el ritmo), permite además establecer fiablemente un vínculo de parentesco entre música y lenguaje ${ }^{11}$. Finalmente, la asociación de ciertas vocalizaciones cuasi-musicales ancestrales con estados emocionales especialmente intensos, relacionados con el deseo, la rivalidad o la consumación sexual, pudo dar lugar a la aparición de una primitiva forma de simbolismo ligada al sexo que, a su vez, pudo servir de base o modelo para el tipo de simbolismo de alcance general propio del lenguaje ${ }^{12}$.

Por otra parte, las razones por las que vio en los pájaros el tipo de organismo realmente modélico para clarificar los orígenes evolutivos del lenguaje tienen que ver con la acumulación de toda una serie de características compartidas realmente llamativas ${ }^{13}$, como el carácter en parte innato y en parte aprendido (o social) de

${ }^{10}$ "As we have every reason to suppose that articulate speech is one of the latest, as it certainly is the highest, of the arts acquired by man, and as the instinctive power of producing musical notes and rythms is developed low down in the animal series, it would be altogether opposed to the principle of evolution, if we were to admit that man's musical capacities have been developed from the tones used in impassioned speech" (Darwin 1871, 638).

11 «We must suppose that the rhythms and cadences of oratory are derived from previously developed musical powers. We can thus understand how it is that music, dancing, song, and poetry are such very ancients arts. We may go even further than this, and [...] believe that musical sounds afforded one of the bases for the development of language» (Darwin 1871, 639).

12 «Primeval man, or rather some early progenitor of man, probably first used his voice in producing true musical cadences, that is in singing, as do some of the gibbon-apes at the present day; and we may conclude from a widely-spread analogy, that this power would have been especially exerted during the courtship of the sexes — would have expressed various emotions, such as love, jealousy, triumph — and would have served as a challenge to rivals. It is, therefore, probable that the imitation of musical cries by articulate sounds may have given rise to words expressive of various complex emotions» (Darwin 1871: 109). "The sexes of many animals incessantly call for each other during the breeding-season; and in not few cases, the male endeavours thus to charm or excite the female. This, indeed, seems to have been the primeval use and means of development of the voice, as I have attempted to show in my "Descent of Man". [...] The habit of of uttering musical sounds was first developed, as a means of courtship, in the early progenitors of man, and this became associated with the strongest emotions of which they were capable, —namely, ardent love, rivalry and triumph. [...] From this fact, and from the analogy of other animals, I have been led to infer that the progenitors of man probably uttered musical tones, before they had acquired the power of articulate speech» (Darwin 1872: 86-88).

13 «The sounds uttered by birds offer in several respects the nearest analogy to language, for all the members of the same species utter the same instinctive cries expressive of their emotions; 
muchas formas de canto, la existencia de una fase temprana equiparable al balbuceo infantil o la existencia en muchos casos de una notable diversidad geográfica (o dialectal) dentro del canto característico de una misma especie ${ }^{14}$. De todos modos, para Darwin era obviamente importante no sólo poder alegar un «organismo modelo» para justificar la viabilidad de la selección sexual como mecanismo capaz de actuar sobre formas de expresión vocal hasta dar gradualmente lugar a una facultad compleja como el lenguaje, sino también señalar un «eslabón» relativamente próximo a los humanos que pudiera hacer creíble la continuidad de la extensísima cadena capaz de unir evolutivamente a éstos con las aves. El organismo al que Darwin confió este importante papel fue el gibón, desde luego, no el primate considerado más próximo al humano en términos evolutivos (lo era el gorila, en tiempos de Darwin), pero sí muy popular por lo elaborado de sus cantos ${ }^{15}$.

Por último, Darwin acompañó con algunas tesis auxiliares su receta evolutiva para el lenguaje, como el papel que pudieron tener la imitación de los sonidos captados en la naturaleza o los efectos hereditarios del uso y desuso de los órganos implicados en el habla ${ }^{16}$. De este modo sería justificable, por ejemplo,

and all the kinds which sing, exert their power instinctively; but the actual song, and even the call notes, are learnt from their parents or foster-parents. These sounds, as Daines Barrington has proved, 'are no more innate than language is in man'. The first attempts to sing 'may be compared to the perfect endeavour in a child to babble'. The young males continue practicing, or as the birdcatchers say, 'recording', for ten or eleven months. The first essays show hardly a rudiment of the future song; but as they grow older we can perceive what they are aiming at; and at last they are said "to sing their song round". Nestlings which have learnt the song of a distinct species, as with the canary-birds educated in the Tyrol, teach and transmit their new song to their offspring. The slight natural differences of songs in the same species inhabiting different districts may be appositely compared, as Barrington remarks, "to provincial dialects"; and the songs of allied, though distinct species may be compared with the languages of distinct races of man" (Darwin 1871,108-109).

14 Véase Aronov et al. (2008), Kroodsma (2004) y Todt y Hultsch (2002) como aproximaciones recientes a estas características del canto de los pájaros.

15 Darwin se ocupa específicamente del canto de los pájaros en el capítulo XIII de The descent of man y comenta brevemente el de los gibones en los capítulos XVIII y XIX de la misma obra. Véase Geissman (2000) para una aproximación actualizada al canto de los gibones.

16 «I cannot doubt that language owes its origin to the imitation and modification of various natural sounds, the voices of other animals, and man's own instinctive cries, aided by signs and gestures» (Darwin 1871, 109); "As the voice was used more and more, the vocal organs would have been strengthened and perfected through the principle of the inherited effects of use; and this would have reacted on the power of speech» (Darwin 1871, 110). 
la inexistencia de habilidades vocales complejas en especies mucho más próximas al hombre que los gibones (acaso efecto del desuso en algún antepasado común) y su reaparición entre los miembros de la especie humana (acaso efecto de la revitalización de una habilidad latente a partir de la observación e imitación de las vocalizaciones realizadas por otras especies ${ }^{17}$. Con todos estos ingredientes a punto, Darwin pudo concluir tranquilizadoramente que «the faculty of articulate speech in itself [does not] offer any insuperable objection to the believe that man has developed from some lower form» (Darwin 1871, 114).

\section{La homología se confirma}

En el año 2001 se hizo pública la identificación del primer gen (FOXP2) inequívocamente relacionado con el desarrollo del lenguaje en los humanos (Lai et al. 2001) ${ }^{18}$. El hallazgo fue posible gracias al descubrimiento algunos años antes de un grupo familiar con una alta prevalencia de un trastorno no exclusiva, pero sí centralmente relacionado con determinados aspectos del lenguaje (control fino de las articulaciones, algunos aspectos de la morfología productiva, etc.). Una pauta de transmisión típicamente mendeliana en esa familia ya había hecho pensar en una mutación en un gen autosómico y dominante (Gopnik 1997), pero sólo la localización de un individuo ajeno a este primer grupo con un tipo de afectación semejante en las funciones lingüísticas y portador de una disrupción en una región del cromosoma 7 ( 7 q31) dio lugar a que se pudiera identificar en todos los afectados originalmente estudiados una mutación característica en la misma región. Desde entonces, se ha acumulado con extraordinaria rapidez información sobre la expresión de ese gen en el cerebro (especialmente en los ganglios basales, el cerebelo y el cortex frontal) ${ }^{19}$, sobre su implicación en el desarrollo de otras estructuras orgánicas (entre otras, el corazón, los pulmones o los intestinos) ${ }^{20}$, sobre la compleja red de regulación en que participa (si bien aún relati-

17 El escocés Lord Monboddo (James Burnet; Origins and progress of language, Vol.I, 1774) sostuvo precisamente que el origen del lenguaje se encontraba en la imitación del canto de los pájaros por parte de los primeros humanos, tesis por cierto conocida por Darwin (1871, 639; nota 40).

18 Véase Benítez Burraco (2009) como síntesis actualizada de toda la información reunida hasta el momento sobre FOXP2, así como las clarificadoras interpretaciones de Longa (2006) y Piattelli-Palmarini \& Uriagereka (en prensa).

19 Ferland et al. (2003), Lai et al. (2003), Liégeois et al. (2003) y Takahashi et al. (2003).

20 Shue et al. (2001). 
vamente escasos) ${ }^{21}$, y sobre su historia evolutiva (los cuales ponen de manifiesto su carácter altamente conservador) ${ }^{22}$.

No obstante, con relación a los intereses de este trabajo, el dato tal vez más sorprendente de todos los que ha producido esta verdadera «industria» en torno a FOXP2 ha sido la constatación de que un gen homólogo (FoxP2) se relaciona en el caso de algunos pájaros con el desarrollo socialmente inducido de los cantos, ya sea durante un período crítico juvenil (como en el caso de los pinzones), ya sea estacionalmente con la llegada de cada nuevo período de celo (como en el caso de los canarios). Se ha verificado, además, que dicha función se relaciona fundamentalmente con los niveles de expresión del gen en la llamada área X, un componente del circuito cerebral anterior vinculado al aprendizaje del canto. Este circuito forma parte de la estructura homóloga en los pájaros a los ganglios basales humanos, en los que, como se señala arriba, también se expresa FOXP2. Además, en estas especies de pájaros el canto sirve como un reclamo por parte de los machos y como un estímulo para la disponibilidad hormonal y comportamental de las hembras con relación al sexo y la maternidad ${ }^{23}$. Se trata, pues, de datos todos ellos importantísimos, porque se corresponden fielmente con los que Darwin sin duda hubiese deseado encontrar para verificar su hipótesis sobre los orígenes evolutivos del lenguaje de haber dispuesto en su día de las teorías y medios técnicos propios de la biología contemporánea.

Pero, ¿podemos realmente concluir a la luz de todos estos datos que la intuición de Darwin se corresponde con un verdadero ejemplo de homología, en el sentido técnico del término? ¿Podemos realmente afirmar que el canto de pájaros como los pinzones o los canarios es una habilidad homóloga al lenguaje de los humanos? Con las debidas precisiones, en las que nos detendremos en los próximos párrafos, la respuesta que a mi juicio cabe dar a estas preguntas es afirmativa.

21 Spiteri et al. (2007).

${ }^{22}$ Enard et al. (2002), Krause et al. (2007), Coop et al. (2008) y Benítez Burraco et al. (2008).

${ }^{23}$ Véase Haesler et al. (2004), Jarvis (2004), Teramitsu et al. (2004), Scharff y Haesler (2005), White y Teramitsu (2006), Haesler et al. (2007), Rochefort et al. (2007), Miller et al. (2008). Véase además Ding y Perkel (2002), Gale y Perkel (2005), Sasaki et al. (2006), Cornil et al. (2008) y Huang y Hessler (2008), que analizan toda una serie de similitudes añadidas desde un punto de vista neuro-químico. 
La primera precisión tiene lógicamente que ver con el hecho de que, en su sentido clásico, la noción de "homología» no se aplica a habilidades, sino a órganos: «the same organ in different animals under every variety of form and function» - de acuerdo con la célebre definición de Owen ${ }^{24}$ - No obstante, la extensión de la idea al estudio comparado y evolutivo de actividades, habilidades o capacidades orgánicas podría basarse en la aplicación de alguna de las siguientes estrategias:

1. derivar la homología de las habilidades de la de las estructuras orgánicas cuya actividad las soporta,

2. basar la homología de las habilidades en el paralelismo de los recursos en que se basa el establecimiento de su identidad durante el desarrollo, o

3. hacer directamente extensible el concepto a la identidad de habilidades.

Desde mi punto de vista, se trata de estrategias no mutuamente excluyentes, en el fondo convergentes y que, en todos lo casos, apuntan a la homología de facto de las habilidades puestas en relación por Darwin. Revisemos brevemente el razonamiento a que da lugar la aplicación de cada una de las estrategias.

La primera estrategia nos mantiene básicamente dentro del concepto tradicional, u oweniano, de homología. Su aplicación en este caso no parece del todo inadecuada teniendo en cuenta, por ejemplo, la implicación tanto en el canto como en el habla de determinadas zonas de los ganglios basales. De acuerdo con la interpretación de Lieberman (2006), los ganglios basales pueden ser descritos desde una perspectiva funcional como una «máquina de secuenciación repetitiva» $y$, atendiendo a ello, pueden ser considerados como uno de los componentes de cualquier sistema de computación basado en la distinción arquitectónica básica entre un «secuenciador» (o "generador de patrones») y un espacio de «memoria de trabajo». Los datos derivados del estudio de la expresión de FOXP2/ FoxP2 pueden interpretarse, por tanto, en el sentido de que las computaciones (o combinaciones) que dan lugar al canto de los pájaros y a las expresiones lingüísticas humanas se basan en el funcionamiento de un mismo tipo de sistema al menos en lo que se refiere al secuenciador, dada la más que razonable homología de las estructuras que lo sustentan en uno y en otro caso.

${ }^{24}$ Lectures on the anatomy and phisiology of the invertebrate animals (1843). 
El aspecto sin embargo problemático de la aplicación de esta estrategia es que no parece existir una correspondencia tan clara, desde un punto de vista anatómico, entre los componentes cerebrales que en cada uno de los casos pudiera servir como espacio de memoria de trabajo. En el caso de los humanos parece bastante razonable suponer que tal función corre a cargo de determinados circuitos corticales, en los que parece centralmente implicada el área de Broca ${ }^{25}$, aunque con proyecciones sobre otras zonas frontales y parieto-temporales ${ }^{26}$. Los pájaros disponen de una estructura cerebral análoga al neo-córtex, pero se trata de un componente de sus cerebros poco desarrollado y más bien parece que la estructura que acoge funciones semejantes a las de aquel es la llamada "cresta ventricular dorsal», considerada homóloga de la amígdala y el claustrum de los mamíferos $^{27}$. En apariencia, pues, estas observaciones rompen la posibilidad de extremar la homología entre canto y lenguaje a la totalidad del sistema computacional que asistiría a ambas habilidades, al menos adoptando una perspectiva anatómica.

De hecho, el que en determinados casos puedan observarse «desviaciones» parciales en la base orgánica de estructuras que, por lo demás, pueden considerarse razonablemente homólogas, es una de las razones por las que Wagner (1989a, 1989b) ha propuesto basar el (por él llamado) «concepto biológico de homología» no en la identidad molecular, tisular o anatómica de las estructuras consideradas, sino en la existencia de mecanismos afines en el establecimiento de su individualidad dentro de los entramados orgánicos a los que cada una de ellas pertenezca. Desde este punto de vista, la propia identificación de la participación de variantes de un mismo gen en el desarrollo de las dos capacidades consideradas (canto y lenguaje), apuntaría directamente a la posibilidad de declararlas homólogas, a pesar de que la expresión de dichas variantes y, más en general, de las cadenas de expresión genética en cuya regulación participan pueda tener lugar en estructuras orgánicas no plenamente coincidentes desde un punto de vista anatómico. El problema, acaso puramente coyuntural, de la aplicación de esta perspectiva es el escaso conocimiento disponible sobre la red de regulación en que FOPX2 se encuentra crucialmente implicada ${ }^{28}$, y la carencia de datos sobre

${ }^{25}$ Véase, además, del ya referido Lieberman (2006), Embick et al. (2000), Grodzinsky (2000), Moro et al. (2001) y Musso et al. (2003).

${ }^{26}$ Como se defiende, por ejemplo, en Aboitiz y García (1997) o Aboitiz et al. (2006).

27 Véanse las detalladas explicaciones al respecto de Striedter (2005).

${ }^{28}$ Véase nota 21. 
la extensión de los paralelismos que pudieran existir con relación a la red correspondiente de FoxP2.

En ausencia de datos que confirmen el alcance de la homología en términos genéticos o, más generalmente, de factores de desarrollo, la tercera de las estrategias apuntadas arriba puede sernos de especial utilidad: es decir, extender directamente el concepto de homología a la identificación de habilidades. El problema al que, en principio, se enfrenta esta estrategia es que parece incongruente con el propio contenido del concepto de homología, que es un concepto que refiere a mecanismos, formas o estructuras y no a la función o utilidad que tales entidades puedan tener. Sin embargo, como explica Love (2007), tal dificultad es superable a poco que entendamos y asumamos la distinción entre, de un lado, la «actividad» propia de una estructura orgánica y, de otro lado, el «uso» que pueda dársele a esa actividad en un determinado contexto orgánico u ambiental ${ }^{29}$. Love argumenta que la primera de estas nociones no plantea ningún conflicto conceptual con relación a la noción de homología, porque la actividad de una estructura es algo que se sigue directamente de la configuración de ésta y que no nos extrae, por tanto, del análisis formal de los organismos y sus partes. Para comprenderlo, conviene pensar en la diferente utilidad (o uso) que en diferentes organismos puede tener un mismo tipo de actividad. En este sentido, el caso del que aquí nos ocupamos aporta una situación especialmente ilustrativa.

Basar en esta estrategia la homología entre el canto de los pájaros y el lenguaje humano implica postular que ambas prácticas se basan en la actividad de sistemas de computación del mismo tipo, que además sabemos también afines, al menos parcialmente, desde los puntos de vista anatómico y del desarrollo. "Computar», en este sentido, no significa otra cosa que la manipulación de símbolos mediante (1) su secuenciación y (2) su eventual mantenimiento en un espacio de memoria de trabajo a efectos de la aplicación de nuevas operaciones sobre las secuencias ya generadas ${ }^{30}$. Nada de esto guarda sin embargo relación con el

29 Véase asimismo Wouters (2003).

30 Aplicar un punto de vista computacional supone, asimismo, considerar todas estas unidades y operaciones pasando por alto su realización última en términos físicos. Véase Fodor (1975), Chomsky (1980) o Pylyshyn (1984) como textos representativos de esta posición sobre la descripción de las operaciones de la mente/cerebro. 
empleo de las representaciones resultantes por parte de otros sistemas mentales ni con su utilidad en las dinámicas homeostáticas de los organismos portadores de los respectivos sistemas. En los pájaros, por ejemplo, dichas representaciones conectan evidentemente con los sistemas senso-motores requeridos para la exteriorización y la apreciación de los cantos. En los humanos, además de conexiones semejantes, las representaciones correspondientes conectan además con diferentes sistemas de representación conceptual, lo que las convierte en expresiones significativas, algo en cambio ajeno a los cantos de los pájaros, que actúan de manera más directa como llamadas de atención no asociadas a contenidos conceptuales $^{31}$. Además, el canto de los pájaros sirve como una forma de reclamo sexual, algo para lo que las conexiones referidas pueden resultar suficientes, mientras que desde el punto de vista del «uso», acaso por la multiplicidad de conexiones que establece con diferentes sistemas conceptuales, no puede decirse que el lenguaje responda a ninguna utilidad característica ${ }^{32}$. Todo esto significa que, aunque desde un punto de vista estrictamente funcional canto y lenguaje no tengan realmente mucho que ver, la idea de que pueda tratarse de habilidades realmente homólogas se sostiene perfectamente atendiendo al punto de vista de la actividad subyacente a los sistemas de computación implicados en cada caso, punto de vista que nos mantiene en realidad dentro de los márgenes del análisis estructural o formal de los sistemas orgánicos ${ }^{33}$.

No es este el lugar para decidir cuál es la base sobre la que en último término debamos fundamentar el concepto biológico de homología. Lo que aquí interesa subrayar es que la aplicación de los puntos de vista en que diferentes autores lo basan actualmente tiene el efecto de fortalecer la idea de que el paralelismo que Darwin intuyó entre el canto de los pájaros y el lenguaje de los humanos se corresponde con un caso en que realmente procede aplicarlo.

31 Véase, por ejemplo, Marler (1998).

32 Sobre la inespecificidad funcional del lenguaje y su significado evolutivo véase Lorenzo (2007).

33 De hecho, otras variantes de FoxP2 han sido puestas en relación con otras habilidades y necesidades de los organismos portadores, como la comunicación ultrasónica que algunas especies de ratones emplean en la comunicación madre-hijo (Shu et al. 2005, Fujita et al. 2008) o la representación espacial mediante ecolocación de algunas especies de murciélagos (Li et al. 2007). A falta de confirmación empírica, podría en todos los casos hablarse de habilidades basadas en la actividad de sistemas de computación homólogos. 


\section{Mejor devo que neo}

Lo expuesto hasta aquí significa que la continuidad que Darwin deseaba establecer entre el lenguaje humano y capacidades propias de otras especies animales puede considerarse razonablemente respaldada por la homología real entre el primero y, por lo menos, el canto de determinadas especies de pájaros. Ahora bien, la confirmación de esta línea de continuidad evolutiva, ¿debe llevarnos necesariamente a conceder que asimismo deben cumplirse los restantes principios del modelo de explicación neo-darwinista ${ }^{34}$, es decir, un gradualismo y una motivación funcional estrictas en los procesos de cambio subyacentes? En esta sección defenderé, siguiendo de cerca ideas desarrolladas en Balari y Lorenzo (2008), que si entendemos que el sistema mental de computación que subyace a la elaboración de las expresiones lingüísticas es el efecto evolutivo de perturbaciones en el desarrollo de las estructuras cerebrales en que dicho sistema se realiza, dichos supuestos pueden ser razonablemente evitados en la explicación. Defenderé, en definitiva, que una reinterpretación de las ideas de Darwin sobre la evolución del lenguaje humano en términos de la biología Evo-Devo ${ }^{35}$ resulta mucho más realista que las que se derivan de una suerte de compromiso u obligación con la ortodoxia Neo-darwinista ${ }^{36}$.

Manteniéndonos en el nivel de análisis computacional referido en la sección anterior, los diferentes autores que han abordado el estudio del canto de algunos pájaros desde esta perspectiva coinciden en atribuirle las características de los «lenguajes de Tipo 3» dentro de la llamada "Jerarquía de Chomsky» de complejidad computacional ${ }^{37}$. La principal característica de estos lenguajes es que la introducción de cada nuevo símbolo en una secuencia se encuentra únicamente condicionada por el símbolo que haya sido introducido con anterioridad. Esto significa, fundamentalmente: (1) que este tipo de lenguajes es únicamente apto para

34 Véase Dawkins (1996) y Dennet (1995) como textos representativos y especialmente clarificadores de las bases del neo-darwinismo.

35 Véase Hall (1999), Carroll (2005) o Minelli (2007) como introducciones de la biología Evo-Devo.

36 Véase Pinker y Bloom (1990) como ejemplo especialmente representativo de esta posición neo (o ultra) darwinista con relación a la evolución del lenguaje.

37 Véase Todt y Hultsch (1998), para el canto del ruiseñor, y Okanoya (2002), para el del gorrión capuchino del japón. Para el estudio de los lenguajes formales, véase Hopcroft y Ullman (1979) y Weir (1994). 
computar relaciones de tipo lineal, y (2) que las exigencias de memoria de trabajo del sistema son en realidad mínimas. Las operaciones propias de los lenguajes de este tipo se pueden replicar a través de los llamados «autómatas de estados finitos», tal como se capta en el siguiente análisis del canto del gorrión capuchino:

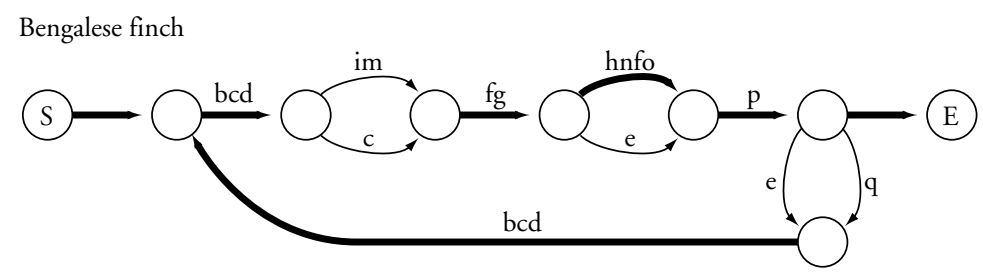

Fig. 1. Representación de las transiciones posibles entre las notas (representadas por letras) que componen el canto de un gorrión capuchino del Japón (Lonchura striata domestica) mediante un autómata de estados finitos. Cada nueva nota que se introduce no responde a otra condición que no sea la de suceder a alguna otra nota en particular. Tomado de Okanoya $(2002,49)$.

El lenguaje humano presenta, en cambio, propiedades formales mucho más sofisticadas, ya que en una misma secuencia siempre se dan, además de relaciones de tipo lineal (unidades que se suceden), relaciones de tipo jerárquico (unidades que se contienen unas a otras) y relaciones a distancia (unidades que remiten a otras no vecinas). Por ejemplo, en una oración relativamente simple como María cree que Juan la desprecia, se manifiestan simultáneamente todos esos tipos de relaciones, tal como trata de captar la siguiente representación:

$$
\left[\mathrm{O}_{1} \text { María }_{\mathrm{i}} \rightarrow \text { cree } \rightarrow\left[\mathrm{O}_{2} \text { que } \rightarrow \text { Juan } \rightarrow \mathrm{la}_{\mathrm{i}} \rightarrow \text { desprecia } \mathrm{O}_{2}\right] \mathrm{O}_{1}\right]
$$

Fig. 2. Representación formal (simplificada) de las relaciones entre los elementos de una oración. Las flechas indican relaciones lineales (precedencia), los corchetes relaciones jerárquicas (incrustación estructural) y los índices relaciones a distancia (en este caso, correferencia).

Todo lo anterior quiere decir que el lenguaje natural humano se corresponde con un «lenguaje de Tipo 1» en la Jerarquía de Chomsky. Esto básicamente significa que se sirve de una pila de memoria de trabajo ampliada relativamente potente, en realidad, un sistema de pilas de memoria simultáneamente activas que permite que cada elemento previamente computado se encuentre accesible para relacionarse con cada nuevo elemento que se introduce en la secuencia. Este estilo de computación se puede replicar mediante un «autómata de pila incrementada». 
Entre los lenguajes de Tipo 3 y los de Tipo 1, ya comentados, la Jerarquía de Chomsky prevé la existencia de un tipo de lenguaje (Tipo 2), con una pila de memoria simple que permite computar relaciones jerárquicas pero no a distancia, y por encima del Tipo 1 sitúa un tipo de lenguaje sin limitaciones de memoria (Tipo 0) y que por tanto sirve para llevar a cabo cualquier tipo de computación. Hauser, Chomsky y Fitch (2002) señalan que los lenguajes de Tipo 2 no parecen corresponderse con las capacidades mentales de ningún organismo ${ }^{38}$, salvo naturalmente los humanos, cuyo lenguaje (de Tipo 1) presupone (y supera) tal régimen computacional ${ }^{39}$. Advierten, no obstante, que resulta necesario explorar dominios como los de la orientación espacial o el cómputo de las relaciones sociales para someter a prueba tal supuesto. Por otro lado, los lenguaje de Tipo 0 deben ser considerados como un «tipo ideal», en el sentido de que la ilimitación memorística que implican los convierte en irrealizables naturalmente.

Balari y Lorenzo (2008) proponen que el acceso a niveles de complejidad computacional creciente ha sido evolutivamente posible gracias a la habilitación y crecimiento de una estructura cerebral capaz de servir como espacio de memoria de trabajo al sistema de computación, que en el caso de los mamíferos se corresponde con el neo-córtex. Defienden, asimismo, que tal crecimiento debe ser puesto en relación con perturbaciones durante el desarrollo individual sobre los factores en que descansa la organización de esa estructura. Y plantean, finalmente, un modelo de evolución que supone (1) la naturalización de la Jerarquía de Chomsky ${ }^{40}$, y (2) su interpretación como un morfo-espacio cognitivo o computacional en que se ve plasmado un conjunto finito, muy limitado y discontinuo correspondiente a los modos de computación accesibles naturalmente. La siguiente figura permite captar la esencia de la propuesta:

38 Gentner et al. (2006) sostienen que el estornino da muestra de una capacidad computacional de este nivel en el reconocimiento de patrones acústicos, el mismo tipo de tarea en que Fitch y Hauser (2004) concluyen que los monos son incapaces de procesar relaciones jerárquicas. Los resultados de este tipo de trabajos han sido, no obstante, muy cuestionados. Véase Perruchet y Rey (2005).

39 Balari y Lorenzo (2009) especulan con la posibilidad de que algunos pájaros hayan accedido a un régimen de computación de Tipo 1, aunque no conectado a habilidades de tipo expresivo. Por ejemplo, los pájaros tejedores hacen nudos para construir sus nidos (véase, por ejemplo, Hansell 2000). El análisis matemático de los nudos revela que su realización implica computaciones equivalentes a las de un lenguaje de Tipo 1. Véase Uriagereka (2007).

${ }^{40}$ Véase, en esta misma línea, Uriagereka (2008). 


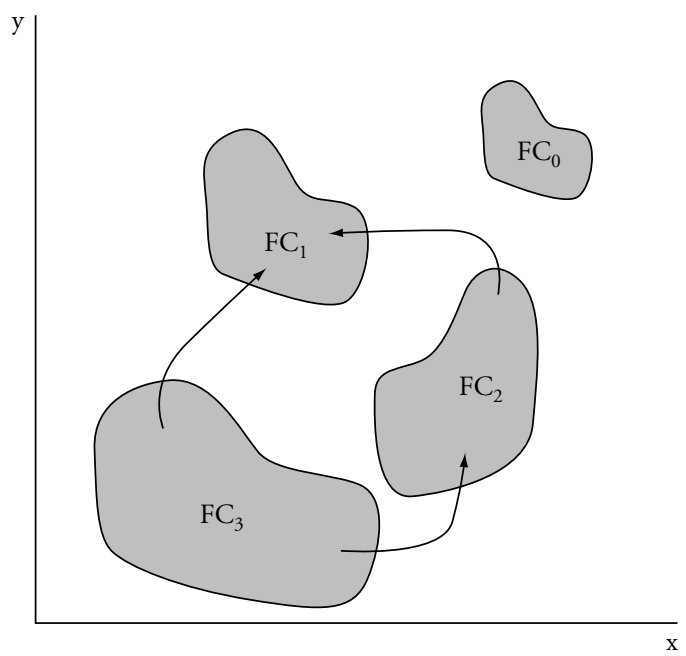

Fig. 3. Morfo-espacio de los fenotipos computacional chomskyanos. Cada uno de estos fenotipos tiene un carácter "discontinuo» (no hay fenotipos con características intermedias) y emerge al alcanzar determinados «valores críticos» los factores o parámetros morfo-genéticos que implantan en el cerebro un sistema de computación. Tomada de Balari y Lorenzo (2009, 30).

Este esquema representa, siguiendo muy de cerca el modelo de «evolución morfológica» de Pere Alberch ${ }^{41}$, un espacio definido (idealmente) por dos factores de desarrollo $(x$ e $y)$. Dentro de este espacio se localizan los fenotipos computacionales $\left(\mathrm{FC}_{3}, \mathrm{FC}_{2}\right.$ y $\mathrm{FC}_{1}$, correspondientes a los tipos 3 a 1 de la Jerarquía de Chomsky) a que da lugar la fijación de diferentes valores para esos parámetros. Nótese que diferentes valores de los factores o parámetros de desarrollo pueden dan lugar a un mismo fenotipo (por ejemplo, $\mathrm{FC}_{3}$ ) y que determinadas modificaciones, no necesariamente radicales, pueden dar lugar, en cambio, a un fenotipo cualitativamente diferente (por ejemplo, $\mathrm{FC}_{2}$ o $\mathrm{FC}_{1}$ ). Las flechas señalan las vías de transición posibles de unos a otros fenotipos (no existe, por tanto, una vía que conduzca naturalmente a $\mathrm{FC}_{0}$ ). Esto significa que una perturbación mínima puede determinar la transición del tipo computacional más básico $\left(\mathrm{FC}_{3}\right)$ a uno cualitativamente más sofisticado $\left(\mathrm{FC}_{2}\right)$ y que una perturbación algo más radical, pero que podría producirse de modo abrupto, puede llevar directamente a un tipo con un nivel de complejidad incluso superior $\left(\mathrm{FC}_{1}\right)$. Por tanto, la transición entre niveles no se plantea como un proceso necesariamente escalonado. Esto supone,

${ }^{41}$ Véase, especialmente, Alberch $(1989,1991)$. 
por ejemplo, que el acceso a un régimen de computación de Tipo 1, como el del lenguaje, podría haber surgido en la naturaleza sin que necesariamente se haya dado una transición evolutiva a través del Tipo 2. Supone, también, que el modelo no requiere de ningún modo que organismos muy próximamente emparentados con los humanos, como los restantes primates superiores, deban disponer de sistemas de computación más próximos al humano que, por ejemplo, el de la aves. El tipo alcanzado por cada organismo será sencillamente el que corresponda a las perturbaciones operadas en su propia historia evolutiva sobre los factores de desarrollo que dan lugar a un sistema cerebral de computación ${ }^{42}$.

Todo lo anterior permite ya articular una respuesta a la pregunta con que se abre esta sección:

- En primer lugar, el modelo presentado parte del supuesto de que la homología entre el canto de los pájaros y el lenguaje humano, postulada por Darwin, es real. La explica apelando a la existencia de un sistema de desarrollo común, es decir, un conjunto de factores de desarrollo compartidos cuya actividad se traduce en la implantación en el cerebro de un sistema de computación. Los valores que en cada caso particular adoptan tales factores explican, por su parte, el tipo o régimen computacional alcanzado por cada organismo.

- En segundo lugar, la continuidad así establecida entre habilidades, en apariencia muy diferentes, compartidas por especies muy distantes en términos de parentesco evolutivo, no requiere sin embargo la postulación de una línea de evolución gradual y acumulativa a lo largo de la cual las propiedades de los sistemas de computación más simples hayan ido adquiriendo las propiedades de los más complejos. En realidad, no existen tipos intermedios entre los modelos de computación chomskyanos. Dentro de cada nivel de jerarquía existen, sí, grados de complejidad creciente que tienen que ver con la potencia de la memoria de trabajo accesible en cada caso ${ }^{43}$. Sin embargo, el paso de uno a otro de los niveles

42 En este sentido, el modelo de Balari y Lorenzo resulta convergente con el de Love (2007), de acuerdo con el cual conceptualizar las homologías en términos de la actividad de las estructuras que la sustentan, en lugar de en términos de la base física de dichas estructuras, facilita la justificación de intermitencias y saltos evolutivos de difícil encaje en el paradigma neo-darwiniano.

43 Véase Pullum y Rogers (2006). 
debe ser visto como un salto que conduce a un régimen de computación en que se hace uso de un tipo de memoria cualitativamente diferenciada. El modelo, además, no requiere una progresión en la jerarquía ni la proximidad o co-presencia en un mismo nivel en consonancia con el parentesco evolutivo entre los organismos. Todo ello resulta acorde con el patrón de intermitencias que parece observarse en la distribución de los niveles de complejidad computacional subyacentes a las habilidades de las diferentes especies animales, especialmente en lo que se refiere a sus comportamientos expresivos.

- Por último, dado el salto cualitativo que supone la elaboración de secuencias con regularidades lineales, estructurales y a distancia (Tipo 1), como las expresiones lingüísticas, frente a la composición de secuencias sujetas a regularidades exclusivamente lineales (Tipo 3), como los cantos de los pájaros, no se sigue de ningún modo de que la función de reclamo sexual atribuida a las últimas haya podido ver incrementada su eficacia mediante la incorporación de las propiedades formales de nivel superior propias de las primeras. Por lo demás, el modelo presentado explica la existencia de cada tipo computacional como efecto de perturbaciones sobre un sistema preexistente de desarrollo que, alcanzados ciertos valores críticos, conducen automáticamente a un nuevo tipo. No cabe, por tanto, conceder a la selección sexual (ni, en realidad, a la selección natural en un sentido más general) la capacidad de ejercer un riguroso control sobre el curso del proceso. Los fenotipos simplemente emergen ${ }^{44}$. Su persistencia tendrá naturalmente que ver con su eficacia dadas unas condiciones ambientales y poblacionales determinadas, pero desde este punto de vista la «selección natural» no es más que un filtro que actúa sobre estructuras ya conformadas ${ }^{45}$.

\section{Conclusión}

La moderna biología evolutiva del desarrollo ha puesto de manifiesto que Darwin, y aún más que él las secuelas neo y ultra darwinistas de su pensamiento, sobrestimaron tanto la capacidad de generación irrestricta y completamente

44 Véase Reid (2007) sobre la noción de «emergencia biólogica».

45 En el sentido, por ejemplo, de Goodwin (1994: 143) o Wagensberg (2004: 125).

46 Fodor y Piattelli-Palmarini (en prensa). 
azarosa de variantes por parte de los organismos como la capacidad de conformación de los fenotipos por parte del ambiente. Cada vez son más las voces científicamente informadas que sostienen que, en lo que se refiere al modelo de evolución mediante selección natural o sexual, Darwin «se equivocó» ${ }^{46}$. De todos modos, resulta suficientemente significativo que doscientos años después de su nacimiento sigamos discutiendo la corrección o el alcance de la corrección de su idea. Por lo demás, ejemplos como el que ha motivado este artículo demuestran de sobra su desbordante imaginación científica. Darwin pertenece a esa lista de biólogos «visionarios» decimonónicos (Goethe, Étienne e Isidore Geoffroy StHilaire, Richard Owen, etc.), muchas de cuyas observaciones han venido siendo confirmadas en los últimos años por las modernas técnicas del análisis genético. No cabe la menor duda de que, sea por la vía de la confirmación, de la corrección parcial o incluso de la refutación, Darwin sigue inspirando algunas de nuestras mejores ideas sobre la naturaleza humana.

\section{Bibliografía}

ABOITIZ, F. y GARCíA, F. 1997. «The evolutionary origin of the language areas in the human brain. A neuroanatomical perspective», Brain Research Reviews 25: 381-396.

Aboitiz, F., García, R., Brunetti, E. y Bosman, C. 2006. «The origin of Broca’s area and its connections from an ancestral working memory network», in GRODZINSKY, Y. y Amunts, K. (eds.), Broca's Region, Nueva York: Oxford University Press: 3-16.

AlBerCH, P. 1989. "The logic of monsters: evidence for internal constraint in development and evolution», Geobios12 (mémoire spécial): 21-57.

- 1991. «Del gen al fenotipo: sistemas dinámicos y evolución morfológica», Revista Española de Paleontología (número extraordinario): 13-19.

Aronov, D., Andalman, A.S. y Fee, M.S. 2008. «A specialized forebrain circuit for vocal babbling in the juvenile songbird", Science 230: 630-634.

BALARI, S. y LORENZO, G. 2009. «Computational phenotypes: Where the theory of computation meets Evo-Devo", Biolinguistics 3: 2-61.

- 2008. «Pere Alberch's developmental morphospaces and the evolution of cognition», Biological Theory, 3(4): 297-304.

Benítez-Burraco, A. 2009. Genes y lenguaje. Aspectos ontogenéticos, filogenéticos y cognitivos, Barcelona: Reverté. 
Benítez-Burraco, A., Longa V. M., Lorenzo, G. y UriagereKa, J. 2008. «Also sprach Neanderthalis... or did she?», Biolinguistics 2.2-3: 225-232.

Burling, R. 2005. The talking ape. How language evolved, Oxford: Oxford University Press.

Carroll, S.B. 2005. Endless Forms Most Beautiful. The New Science of Evo-Devo, New York: Norton \& Company.

Chomsкy, N.1980. Rules and representations, New York: Columbia University Press.

Coop, G., Bullaughey, K., Luca, F. y Przeworski, M. 2008. «The timing of selection at the human FOXP2 gene», Molecular Biology and Evolution 25: 1257-1259.

Cornil, C.A., Castelino, C.B. y BALl, G.F. 2008. «Dopamine binds a-adrenergic receptors in the song control o zebra finches (Taeniopygia guttata)", Journal of Chemical Neuroanatomy 35: 202-215.

DARWIN, CH. 1871. The descent of man, and selection in relation to sex, [Second edition, 1879], London: John Murray [citado por la edición de James Moore y Adrian Desmond, London: Penguin Classics, 2004].

- 1872. The expression of the emotions in man and animals, [Second edition, 1890], London: John Murray [citado por la edición de Joe Cain y Sharon Messenger, London: Penguin Classics, 2009].

DaWKINS, R. 1996. Climbing Mount Improbable, New York: W.W. Norton and Co.

DEACON, T. 1997. The symbolic species. The co-evolution of language and the human brain, London: Penguin.

Dennett, D.C. 1995. Darwin's Dangerous Idea, New York: Simon \& Schuster

Ding, L. y PERKEL, D.J. 2002. "Dopamine modulates excitability of spiny neurons in the avial basal ganglia», Journal of Neuroscience 22: 5210-5218.

Embick, D., Marantz, A., Miyashita, Y., O’Neil, W. y SaKai, K.L. 2000. «A syntactic specialization for Broca's area», PNAS USA 97: 6150-6154.

Enard, W., Przeworki, M., Fischer, S.E, Lai, C.S., Wiebe, V., Kitano, T., MonaCO, A.P. y PÄÄBO, S. 2002. «Molecular evolution of FOXP2, a gene involved in speech and language», Nature 418: 868-872.

Ferland, R.J., Cherry, T.J., Preware, P.O., Morrisey, E.E. y Walhs, Ch.A. 2003. "Characterization of FOXP2 and FOXP1 ARNm and protein in the developing and mature brain", Journal of Comparative Neurology 460: 266-279. 
FitCH, T. y Hauser, M.D. 2004. "Computational constraints on syntactic processing in a nonhuman primate», Science 303: 377-380.

FODOR, J. 1975. The language of thought, New York: Thomas Y. Crowell Co.

Fodor, J. y Piattelli-Palmarini, J. en prensa. What Darwin got wrong, New York: Farrar, Straus \& Giroux.

Fujita, E., Tanabe, Y., Shiota, A., Ueda, M. Suwa, K., Momoi, M.Y. y Momoi, T. 2008. "Ultrasonic vocalization impairment of Foxp2 (R552H) knockin mice related to speech-language disorder and abnormality of Purkinje cells», PNAS USA 105: 3117-3122.

Gale, S.D. y PERKEL, D.J. 2005. «Properties of dopamine release and uptake in the songbird basal ganglia», Journal of Neurophysiology 93: 1871-1879.

GEISSMANN, T. 2000. "Gibbon songs and human music from an evolutionary perspective», en Wallin, N.L. Merker, B. y Brown, S. (eds.), The Origins of Music, Cambridge, MA: The MIT Press: 103-123.

Gentner, T.Q., Fenn, K.M., Margoliash, D. y Nusbaum, H. 2006. «Recursive syntactic pattern learning by songbirds», Nature 440: 1204-1207.

Goodwin, B. 1994. How the leopard changed its spots. The evolution of complexity, London: Phoenix.

GOPNIK, M. (ed.). 1997. The inheritance and innateness of grammars, Oxford: Oxford University Press.

GrodZINSKY, Y. 2000. "The neurology of syntax: language use without Broca's area», Behavioral and Brain Sciences 23: 1-71.

Haesler, S., Rochefort, C., Georgi, B. Licznerski, P., Osten, P. y Scharff, C. 2007. «Incomplete and inaccurate vocal imitation after knockdown of FoxP2 in songbird basal ganglia nucleus Area X», PLoS Biology 5: e321.

Haesler, S., Wada, K., Nshdejan, A., Morrisey, E., Lints, T., Jarvis, E. D. y Scharff, C. 2004. "Foxp2 expression in avian vocal learners and non-learners», The Journal of Neuroscience 24: 3164-3175.

Hall, B.K. 1999. Evolutionary developmental biology. Second edition, Dordrecht: Kluwer Academic.

HANSELl, M.K. 2000. Bird nests and construction behaviour, Cambridge: Cambridge University Press.

Hauser, M.D., Chomsky, N. y Fitch, T. 2002. «The faculty of language: What is it, who has it, and how did it evolve?», Science 298: 1569-1579. 
Hopcroft, J.E. y UlLman, J.D. 1979. Introduction to automata theory, languages, and computation, Reading, MA: Addison Wesley.

HuANG, Y.C. y HeSSLER, N.A. 2008. «Social modulation during songbird courtship potentiates midbrain dopaminergic neurons», PloS ONE 3: e3281.

JARVIS, E.D. 2004. «Learned bird song and the neurobiology of human language», Annuary of the New York Academy of Sciences 1016: 749-777.

JeSPERSEN, O. 1922. Language. Its nature, development and origin, London: George Allen \& Unwin Ltd..

Krause, J., Lalueza-Fox, C., Orlando, L., Enard, W., Green, R. E., Burbano, H. A., Hublin, J.-J., Hänni, C., Fortea, J., de la Rasilla, M., Bertranpetit, J., ROSAS, A. y PÄÄBO, S. 2007. "The derived Foxp2 variant of modern humans was shared with Neandertals», Current Biology 17: 1908-1912.

KroOdSMA, D. 2004. «The diversity and plasticity of birdsong», in MerLER, P.R. y SLABBEKOORN, H. (eds.), Nature's music. The science of birdsong, San Diego, CA: Elsevier: 108-131.

Lai, C.S., Fisher, S.E., Hurst, J.A., Vargha-Khandem, F. y Monaco, A.P. 2001. «A forkhead-domain gene is mutated in a severe speech and language disorder», Nature 413: 519-523.

LaI, C.S., GerRelli, D., Monaco A.P., Fisher, S.E., y CoPP, A.J. 2003. «FOXP2 expression during brain development coincides with adult sites of pathology in a severe speech and language disorder», Brain 126: 2455-2462.

Li, G., Wang, J., Rossiter, S. J., Jones, G. y Zhang, S. 2007. «Accelerated FoxP2 evolution in echolocating bats», PloS ONE 2: e900.

LieBerman, P. 2006. Toward an evolutionary biology of language, Cambridge, MA: Harvard University Press.

LiéGeois, F., BadelWeg, T., Connelly, A., Gadian, D.G., Mishrin, M. y VarghaKHADEM, F. 2003. «Language fMRI abnormalities associated with FOXP2 gene mutation", Nature Neuroscience 6: 1230-1237.

LONGA, V.M. 2006. «Sobre el significado del descubrimiento del gen FOXP2», Estudios de Lingüistica. Universidad de Alicante 20: 177-207.

LORENZO, G. 2006. El vacío sexual, la tautología natural y la promesa minimalista. Ensayos de biolingüistica, Madrid: Antonio Machado Libros. 
— 2007. «Es el lenguaje una adaptación», en Coca, J.R. (coord.), Varia biologica. Filosofía, ciencia y tecnología. Colección Contextos 17, León: Centro de Estudios Metodológicos e Interdisciplinares/Univ. de León: 189-219.

LOVE, A.C. 2007. «Functional homology and homology of function: biological concepts and philosophical consequences». Biology and Philosophy 22: 691-708.

MARLER, P. 1998. «Animal communication and human language», en JABLONSKI, N.G y AiELLO, L.C. (eds.), The origin and diversification of language, San Francisco: Memoirs of the California Academy of Sciences 24: 1-19.

MiLler, G. 2000. The mating mind. How sexual choice the evolution of human nature, New York: Anchor Books.

Miller, J.E., Spiteri, E., Condro, M.C., Dosumu-Johnson, R.T., Geschwind, D.H. y White, S.A. 2008. «Birdsong decreases protein levels of FoxP2, a molecule required for human speech», Journal of Neurophysiology 100: 2015-2025.

Minelli, A. 2007. Forme del divenire, Torino: Einaudi [citado por la versión en inglés, Forms of becoming. The evolutionary biology of development, Princeton and Oxford: Princeton University Press, 2009].

Mithen, S. 2006. The singing Neanderthals. The origins of music, language, mind, and body, Cambridge, MA: Harvard University Press.

Moro, A., Tettamanti, M., Perani, D., Donati, C., Cappa, S.F. y Fazio, F. 2001. "Syntax and the brain: disentangling grammar by selective anomalies», NeuroImage 13: $110-118$.

Musso, M., Moro, A., Glauche, V., Rijntjes, M., Reichenbach, J., Büchel, Ch. y WeILlER, C. 2003. «Broca's area and the language instinct». Nature Neuroscience 6: 774-781.

OKANOYA, K. 2002. «Sexual display as a syntactic vehicle: The evolution of syntax in birdsong and human language through sexual selection», en WRAY, A. (ed.), The transition to language, Oxford: Oxford University Press: 46-64.

Perruchet, P. y Rey, A. 2005. «Does the mastery of center-embedded linguistic structures distinguish humans from nonhuman primates?», Psychonomic Bulletin \& Review 12: 307-313.

Piattelli-Palmarini, M. y Uriagereka, J. en prensa. «A geneticist's dream, a linguist's nightmare: The case of FOXP2», en Di SCIUllo, A.M. y AGUero, C. (eds.), Biolinguistic investigations, Cambridge, MA: The MIT Press. 
Pinker, S. y BloOm, P. 1990. «Natural language and natural selection», Behavioral and Brain Sciences 13: 707-784.

Pullum, G.K. y Rogers, J. 2006. «Animal pattern-learning experiments: some mathematical background», ms., Radcliffe Institute for Advanced Study, Harvard University.

Pylyshyn, Z. 1984. Computation and cognition: Toward a foundation for cognitive science, Cambridge, MA: The MIT Press.

REID, R.G.B. 2007. Biological emergences. Evolution by natural experiment, Cambridge, MA: The MIT Press.

Ridley, M. 1993. The Red Queen. Sex and the evolution of human nature, New York: Perennial.

Rochefort, C., He, X., Scotto-Lomassese, S. y Scharff, C. 2007. «Recruitment of FoxP2-expressing neurons to area $\mathrm{X}$ varies during song development», Developmental Neurobiology 67: 809-17.

RupKe, N.A. 1994. Richard Owen. Victorian naturalist, New Haven \& London: Yale University Press.

SASAKI, A., SotnikOVA, T.D., GAInetdinOV, R.R. y JaRVIS, J.D. 2006. «Social contextdependent singing-regulated dopmaine», Journal of Neuroscience 26: 9010-9014.

SCHARFF, C. y HAESLER, S. 2005. «An evolutionary perspective on FoxP2: strictly for the birds?», Current Opinion in Neurobiology 15: 694-703.

Shu, W., Cho, J. Y., Jiang, Y., Zhang, M., Weisz, D., Elder, G. A., Schmeildler, J., De Gasperi, R., Gama Sosa, M. A., Rabidou, D., Santucci, A. C., Perl, D., Morrosey, E. y BuXBaum, J. D. 2005. "Altered ultrasonic vocalization in mice with a disruption in Foxp2 gene», PNAS USA 102: 9643-9648.

SHU, W., YANG, H., ZHANG, L., LU, M.M. y MORRISEY, E.E. 2001. «Characterization of a new family of wingedhelix/forkhead (Fox) genes that are expressed in the lung and act as transcriptional repressors», Journal of Biological Chemistry 276: 2748827497.

Spiteri, E., Konopka, G., Coppola, G., Bomar, J., Oldham, M. OU, J., Vernes, S. C., Fisher, S. E., Ren, B. y Geschwind, D. H. 2007. «Identification of the transcriptional targets of FOXP2, a gene linked to speech and language, in developing human brain", American Journal of Human Genetics 81: 1144-1157.

Striedter, G.F. 2005, Principles of brain evolution, Sunderland, MA: Sinauer. 
Takahashi, K., LiU, F.-CH., Hirokawa, K. y Takahashi, H. 2003. «Expression of FOXP2, a Gene Involved in Speech and Language, in the Developing and Adult Striatum», Journal of Neuroscience Research 73: 61-72.

Teramitsu, I., Kudo, L.C., London, S.E, Geschwind, D.H. y White S.A. 2004. «Parallel FoxP1 and FoxP2 expression in songbird and human brain predicts functional interaction», Journal of Neuroscience 24: 3152-3163.

TodT, D. y Hultsch, H. 1998. «How songbirds deal with large amounts of serial information: retrieval rules suggest a hierarchical song memory», Biological Cybernetics 79: 487-500.

— 2002. "Social aspects of vocal learning and song use in birds», Proceedings of the $23^{\text {rd }}$ International Ornithological Congress, Beijing. Vol. 3: 112-118.

URIAGEREKA, J. 2007. "What songbirds, dancing, and knot-tying can tell us about why we speak», Seedmagazine.com [september 25].

— 2008. "Desperately evolving syntax», in SMITH, A.D.M., SMITH, K. y FERRER I CANCHO, R. (eds.), The evolution of language, Singapur: World Scientific: 331-337.

WAGENSBERG, J. 2004. La rebelión de las formas o cómo perseverar cuando la incertidumbre aprieta, Barcelona: Tusquets.

WaGner, G.P. 1989a. "The biological homology concept», Annual Review of Ecology and Systematics 20: 51-69.

- 1989b. «The origin of morphological characters and the biological basis of homology», Evolution 43: 1157-1171.

WeIR, D. 1994. «Linear iterated pushdowns», Computational Intelligence 10: 431-439.

White, S. y TERAMitsu, I. 2006. «FoxP2 regulation during unidirected singing in adult songbirds», Journal of Neuroscience 26: 7390-7394.

Wouters, A. 2003. «Four notions of biological function», Studies in History and Philosophy of Biological and Biomedical Sciences 34: 633-668.

Recibido: 20/08/2009

Revisado: 26/09/2009 
